Setting a New Standard in the Analysis of Binary Stars

K. Pavlovski, A. Tkachenko and G. Torres (eds)

EAS Publications Series, 64 (2013) 47-54

\title{
TEMPERATURE AND GRAVITY SCALES IN HOT STARS
}

\author{
M.-F. Nieva ${ }^{1,2}$
}

\begin{abstract}
Precise determinations of effective temperatures and surface gravities are mandatory to derive not only reliable chemical abundances, but also parameters like distances, masses, radii and luminosities in OB stars. We have previously determined atmospheric parameters of 30 well-studied OB main sequence to giant stars via multiple ionization equilibria in NLTE, reaching uncertainties as low as $\sim 300 \mathrm{~K}$ for effective temperature and $\sim 0.05$ dex for surface gravity. Based on the comparison of our spectroscopic parameters and reddening-independent quantities of the Johnson and Strömgren photometric systems, temperature and gravity calibrations are proposed to different photometric indices depending on the luminosity class. With these calibrations, effective temperatures can be determined at a precision of $\sim 400 \mathrm{~K}$ for luminosity classes III/IV and of $\sim 800 \mathrm{~K}$ for luminosity class V. Surface gravities can reach internal uncertainties as low as $\sim 0.08$ dex. Our uncertainties are smaller than typical differences among other methods in the literature, which reach values up to $\pm 2000 \mathrm{~K}$ for temperature and \pm 0.25 dex for gravity, and in extreme cases, $+6000 \mathrm{~K}$ and \pm 0.4 dex, respectively.
\end{abstract}

\section{Introduction}

The spectroscopic group OB refers to stars with spectral types between $\sim 09$ and B2. The lower boundary of this group (B2) corresponds to lower mass and upper age limits of $\sim 8 M_{\odot}$ and $30 \mathrm{Myr}$, respectively, and the occurrence of core-collapse supernovae. OB stars delineate the spiral structure of our Milky Way and play an important role in cosmic evolution. They dominate the light of spiral and irregular galaxies and influence the interstellar medium, star formation and galactic evolution through their ultraviolet radiation, winds and supernova explosions.

\footnotetext{
${ }^{1}$ Dr. Karl Remeis-Observatory \& ECAP, University of Erlangen-Nuremberg, Sternwartstr. 7, 96049 Bamberg, Germany

${ }^{2}$ Institute for Astro- and Particle Physics, University of Innsbruck, Technikerstr. 25/8, 6020 Innsbruck, Austria
} 
In the present work, we mainly focus on the temperature and gravity scales of OB stars in the Main Sequence and Giant phases: the core H-burning phases. We investigate whether our recent improvements made in the acquisition of observational data, calculation of more realistic theoretical spectra and in the spectral analysis methodologies, affect the overall effective temperature and surface gravity scales of those objects. A quantitative spectroscopic analysis for a representative sample of 30 stars in the solar neighbourhood has been performed by Nieva \& Przybilla (2012), hereafter Paper I), Nieva \& Simón-Díaz (2011), Paper II) and Briquet et al. (2011), Paper III), where multiple independent ionization equilibria were evaluated iteratively together with fits to Balmer lines in NLTE in order to reproduce the whole observed spectrum in the optical. The analysis resulted in a unique set of atmospheric parameters at high precision and accuracy for each star. Based on the previous work, we provide here a summary of new calibrations to estimate temperatures and gravities of $\mathrm{OB}$ stars from photometric quantities and their spectral classification. For more details see Nieva (2013).

\section{The star sample and observations}

Our star sample covers spectral types from B3 to O9. The stars are located between the Zero Age and the Terminal Age Main Sequence in the Hertzsprung-Russell diagram. Their temperatures range from $\sim 16000$ to $34000 \mathrm{~K}$, the logarithmic surface gravities from $\sim 3.6$ to 4.3 (cgs units) and the masses from $\sim 5$ to $20 M_{\odot}$. Details of the star sample like spectral types, photometric quantities, atmospheric parameters derived in Papers I-III can be found in Table 1 of Nieva (2013).

We aimed at selecting single stars, as second light from a companion of a similar spectral class distorts the ratio of line-to-continuum fluxes. Thus, objects in SB1 systems with much fainter companions can therefore still qualify as targets, because the contribution of the secondary star in optical light $(U B V, u v b y)$ is marginal. Individual components in a visual binary are also considered as targets.

The spectral analysis was based on high-resolution échelle spectra at very high signal-to-noise $(\mathrm{S} / \mathrm{N})$ ratio - ranging from 250 up to over 800 in $B$ - and wide wavelength coverage. The spectra were obtained with FEROS on the ESO $2.2 \mathrm{~m}$ telescope in La Silla/Chile, $R=\lambda / \Delta \lambda \approx 48000$, FOCES on the $2.2 \mathrm{~m}$ telescope at Calar Alto/Spain, $R \approx 40000$, the ELODIE échelle spectrograph on the $1.93 \mathrm{~m}$ telescope of the Observatoire de Haute-Provence/France, $R \approx 42000$, and FIES mounted at the $2.5 \mathrm{~m}$ Nordic Optical Telescope (NOT) in El Roque de los Muchachos observatory on La Palma (Canary Islands, Spain), $R \approx 46000$.

\section{Spectroscopic stellar parameter determination in NLTE}

All atmospheric parameters were determined simultaneously via line-fitting of synthetic spectra in NLTE to the observed data, as described in Papers I-III. Atmospheric parameters are the effective temperature $T_{\text {eff }}$, surface gravity $\log g$, microturbulence $\xi$, projected rotational velocity $v \sin i$ and macroturbulence $\zeta$. 
The spectral analysis was based on a grid of synthetic spectra in NLTE computed with the codes Detail and Surface (Giddings 1981; Butler \& Giddings 1985) on prescribed LTE atmospheric structures calculated with ATLAS9 (Kurucz 1993b). Model atoms for $\mathrm{H}, \mathrm{He}, \mathrm{C}, \mathrm{N}, \mathrm{O}, \mathrm{Ne}, \mathrm{Mg}, \mathrm{Si}$ and Fe for different ionization stages were employed. Opacities due to hydrogen and helium and the individual metals were considered in NLTE, and line blocking was accounted for in LTE via Kurucz' Opacity Distribution Functions (Kurucz 1993a). This approach provides an efficient way to compute realistic synthetic spectra when the atmosphere is close to LTE. In particular, it is equivalent to full NLTE model atmosphere calculations for late O-type to early B-type stars with weak winds (Nieva \& Przybilla 2007; Przybilla et al. 2011).

The spectral analysis follows the methodology introduced by Nieva (2007) and applied for H/He and C by Nieva \& Przybilla $(2007,2008)$ and for other elements by Przybilla et al. (2008). Stellar parameters were determined through ionization equilibria for different chemical species and Balmer lines fits. The reliability of our temperature determinations and the minimisation of systematic uncertainties are confirmed by simultaneous agreement of several independent indicators: ionization equilibria, fits to Balmer lines and fits to the spectral energy distributions from the UV to the IR. Furthermore, our surface gravities are also confirmed by simultaneous match of ionization equilibria, fits to Balmer lines and the agreement of spectroscopic distances with Hipparcos distances (see Paper I). Note that every model atom for NLTE calculations is independent from each other, hence multiple ionization equilibria render 4-5 independent indicators for temperature and gravity determinations.

\section{Temperature and gravity scales}

We investigate whether new temperature and gravity scales to the $Q$-index ${ }^{3}$ in the broad-band Johnson $U B V$-system, the $[u-b]$ and $\left[c_{1}\right]$-parametes ${ }^{4,5}$ from the narrow-band Strömgren $u b v y$-system and $\beta^{6}$ can be established based on our previously derived spectroscopic parameters. Our atmospheric parameters offer a unique set of input data for building such scales: uncertainties are as low as $\sim 1-$ $2 \%$ for effective temperatures and $\sim 10 \%$ for surface gravities. This is in contrast to uncertainties of $\sim 5-10 \%$ and $\sim 25 \%$, respectively, using standard methods. Typical uncertainties for effective temperature given in the literature are $\leq 3-5 \%$, however these do not take into account the large discrepancies - up to $\sim 6000 \mathrm{~K}$ - that exist between different temperature determination methods. Error estimates of up to $40-60 \%$ in surface gravity are also found in the literature. Likewise, the most accurate photometric data available in the literature are adopted for this study. The compilation of photometric data is as homogeneous as possible, however for some

\footnotetext{
${ }^{3} Q=(U-B)-0.72(B-V)$.

${ }^{4}[u-b]=(u-b)-1.5(b-y)=c_{1}+2 m_{1}+0.5(b-y)$.

${ }^{5}\left[c_{1}\right]=c 1-0.2(b-y)$.

${ }^{6} \beta$ is a measure of the stellar $\mathrm{H} \beta$ line intensity.
} 

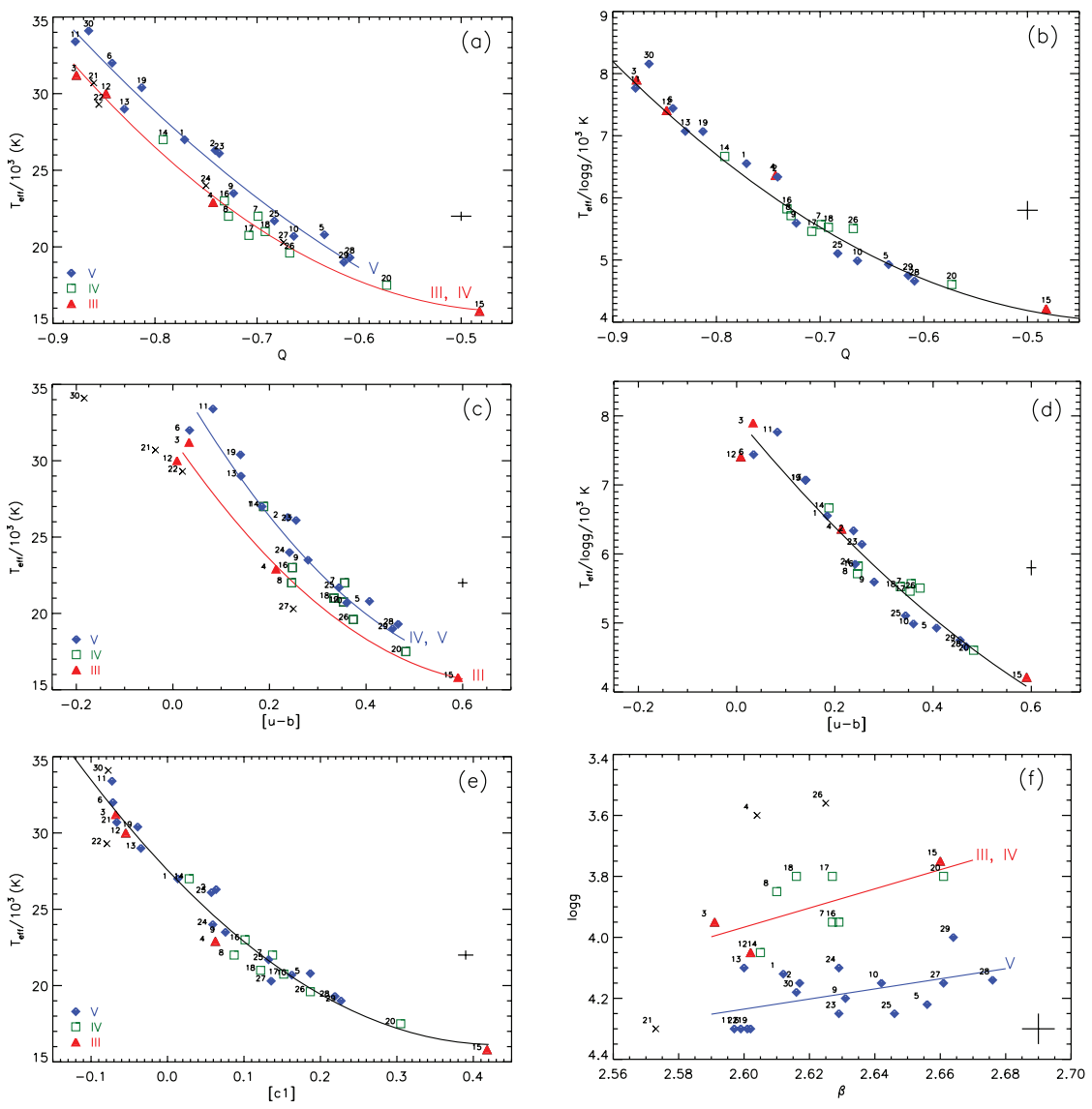

Fig. 1. Functional relationships for the dependence of $T_{\text {eff }}$ (left) and $\log g$ (right panels) on the photometric parameters $Q(\mathrm{a}, \mathrm{b}),[u-b](\mathrm{c}, \mathrm{d}),\left[c_{1}\right]$ (e) and $\beta$ (f). Symbols correspond to the sample stars and curves are polynomial fits to the data for different luminosity classes, encoded according to the legend. A few stars have been excluded from the fits (crosses). Typical error bars are indicated. See the text for details. From Nieva (2013).

objects, data are adopted from different sources. In addition, re-examinations of spectral types and luminosity classes were performed for the sample stars.

Empirical relationships of atmospheric parameters and photometric indices based on data of our star sample are presented here. Note that the star sample does not cover uniformly the parameter space under consideration, therefore the relationships can be refined when adopting a more complete sample. Figure 1a shows the relation between our spectroscopic temperatures and $Q$, while Figure $1 \mathrm{~b}$ displays the behavior of $T_{\text {eff }} / \log g$ vs. $Q$. The data are indicated by symbols and the polynomial fits by full lines, encoded for different luminosity classes as shown in the legend. Four objects of luminosity class V had to be excluded from 
the analysis - marked with a cross in Figure 1a. These stars are located in the Orion OB association, in a region of dense nebulosity and anomalous reddening. The distinction of different luminosity classes reveals tighter trends of $T_{\text {eff }} v s$. $Q$ than previously reported. The trends are consistent with those found by Nieva \& Przybilla (2008) for a sub-set of stars. In the present work, the polynomial fits for luminosity classes III and IV converge into a single curve and the scale for luminosity class $\mathrm{V}$ is $\sim 1500$ to $2000 \mathrm{~K}$ hotter. The minimisation of uncertainties in our spectroscopic parameter determination and the high accuracy of the photometric data allow clear trends for different luminosity classes to be distinguished. The relationship between the temperature and $Q$ for different luminosity classes, i.e., different evolutionary stages, displayed in Figure 1a can be expected by the fact that stars closer to the Zero Age Main Sequence are more compact and bluer, therefore hotter than the more evolved giants at similar masses.

In contrast, the ratio $T_{\text {eff }} / \log g$ vs. $Q$ does not show a dependence on the luminosity class (Fig. 1b), since all three polynomial fits converge approximately to a single solution. Therefore, we adopt one fit to the whole sample (except for the excluded stars), indicated by the thick black curve.

Figures 1c,d show the relationship between $[u-b]$ and our spectroscopic temperatures and $T_{\text {eff }} / \log g$, respectively. The data are encoded like in Figures 1a,b. Similarly to the case of the $Q$-parameter, trends of $T_{\text {eff }} v s$. $[u-b]$ are found for different luminosity classes. In this case, the polynomial fits for luminosity classes IV and $\mathrm{V}$ converge into a single relation, while the scale for luminosity class III is $\sim 2000$ to $4000 \mathrm{~K}$ cooler. In addition, $T_{\text {eff }} / \log g$ vs. $[u-b]$ does also not indicate a dependence on the luminosity class (Fig. 1d).

The Strömgren parameters $\left[c_{1}\right]$ and $\beta$ have been defined as tools to estimate $T_{\text {eff }}$ and $\log g$ in early-type stars. A single trend for the effective temperature vs. [c1] (Fig. 1e) and two curves for $\log g$ vs. $\beta$ (Fig. 1f) are found. For the latter, the relationship for luminosity class $\mathrm{V}$ is $\sim 0.3$ dex higher than for III/IV. Stars \#4 and \#26 have been excluded from the fits because their gravities are too low - therefore the stars are too evolved - in comparison with the rest of the stars of luminosity class III/IV. Star \# 21 is also excluded, as there is a large nebular emission feature centered on $\mathrm{H} \beta$, that causes a significantly reduced value of the $\beta$-index. As the parameter range of interest is not uniformly sampled by the observations, a further addition of few hotter stars with low surface gravities might produce a flattening of the slope for luminosity class V in Figure 1f, which remains to be investigated.

\section{Our calibrations $v s$. photometric calibrations in the literature}

Our $T_{Q}$-scales derived from multiple ionization equilibria are compared in Figure 2a to photometric calibrations of Daflon et al. (1999) for luminosity class V and of Lyubimkov et al. (2002) for $\log g=3.48$ and 4.02. In addition, our $T_{[u-b]^{-}}$ scales are compared to that of Napiwotzki et al. (1993) in Figure 2b. Our scales tend to be hotter at higher temperatures than the previous photometric scales. Our $T_{Q}$ for luminosity class $\mathrm{V}$ (upper solid curve, blue in the online version) is 

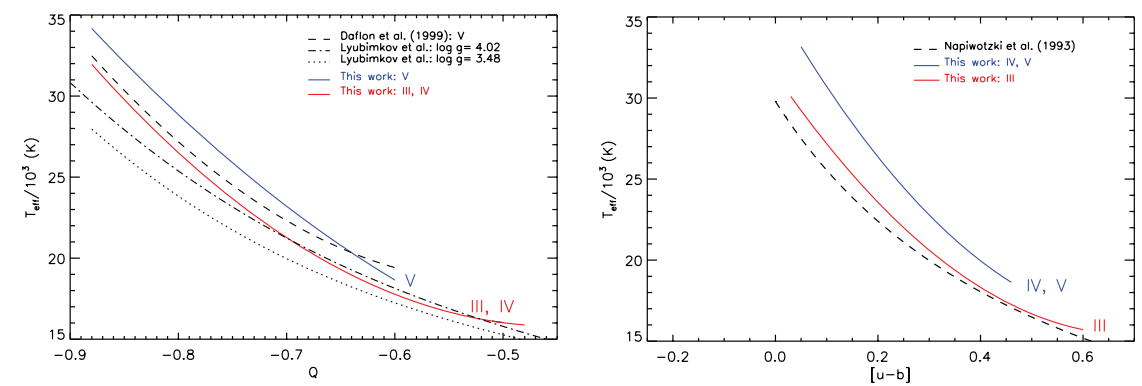

Fig. 2. Left panel: comparison of our $Q$-based temperature relation with those proposed by Daflon et al. (1999) and Lyubimkov et al. (2002). Right panel: comparison of our $[u-b]$-based temperature relation with that from Napiwotzki et al. (1993). See the text for a description. From Nieva (2013).

$\sim 2000 \mathrm{~K}$ hotter than that proposed by Daflon et al. (1999) at the highest temperature, the difference decreasing until both scales cross each other at $\sim 21000 \mathrm{~K}$, and remain similar at slightly lower $T_{\text {eff }}$. The comparison to Lyubimkov et al. (2002) has to be done more cautiously because our sample has a variety of surface gravity values. Only four stars have similar gravities to 4.00: \#3 (luminosity class III), 7 (IV), 16 (IV) and 29 (V). We compare their upper curve (dashed dotted line) to our lower curve (solid line, red in the online version). Our relation is $\sim 2000 \mathrm{~K}$ hotter than theirs at the highest temperatures and both practically coincide below $21000 \mathrm{~K}$.

Figure $2 \mathrm{~b}$ shows the comparison of our $T_{[u-b]}$ to the calibration proposed by Napiwotzki et al. (1993) in their Equation (9). Their and our curve for luminosity class III practically coincide below $19000 \mathrm{~K}$, and the smallest discrepancy to our $\mathrm{IV}, \mathrm{V}$ relation is $\sim 1500 \mathrm{~K}$ at $T_{\text {eff }} \sim 18000 \mathrm{~K}$. In contrast, our scales are steeper than their curve, resulting in larger differences at higher $T_{\text {eff }}$, with maximum values of $\sim 1500 \mathrm{~K}$ for luminosity class III and $\sim 6000 \mathrm{~K}$ for IV,V.

\section{Our parameters $v s$. other determinations for individual stars}

Besides the photometric temperature scales discussed above, other determinations of temperature and surface gravity are found in the literature for several stars of the sample. We have compared our data with determinations by Kilian et al. (1991), Cunha \& Lambert (1992) and Morel \& Butler (2008) for individual stars based on different observed spectra, stellar atmosphere codes and analyses techniques. In general, their temperatures tend to be lower on the upper temperature range and higher or similar on the lower temperature range, see Nieva (2013).

In contrast, determinations of atmospheric parameters by Simón-Díaz (2010) based on the same observational data but on another stellar atmosphere code and a different analysis technique, render very similar values than those derived by us. Stellar parameters were determined in that work spectroscopically by fitting the 


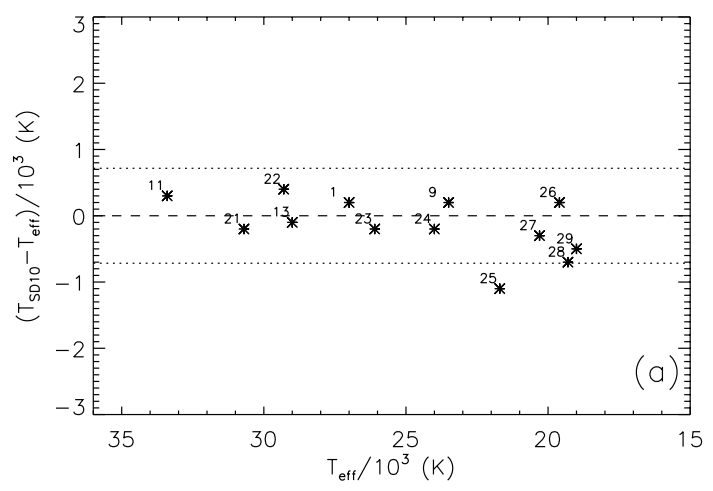

Fig. 3. Differences between $T_{\text {eff }}$ derived by Simón-Díaz (2010) and our spectroscopic values. Dotted lines indicate the averaged internal uncertainties of our $Q$-calibrations.

Balmer lines and matching helium and/or silicon ionization equilibria. Figure 3 illustrates the differences between temperatures derived by Simón-Díaz (2010) and our spectroscopic $T_{\text {eff }}$. Dashed lines indicate full agreement between his determinations and our values, and dotted lines denote $\Delta T_{Q}(700 \mathrm{~K})$, as a reference to our photometric scale. Note that the uncertainty in our spectroscopic $T_{\text {eff }}$ is $\sim 300 \mathrm{~K}$.

The spectrum synthesis in Simón-Díaz (2010) was performed with the unified atmosphere code FASTwIND (Puls et al. 2005), that models the stellar atmosphere and wind simultaneously in NLTE. Despite the different atmospheric structure, input atomic data and line-formation computations, the temperatures and gravities derived in this work agree overall very well with our determinations: the differences lie within the $1 \sigma$-uncertainty of the $Q$-calibrations for most stars.

\section{Our recommended parameter determination}

The excellent agreement between our and Simón-Díaz (2010) temperatures lead us to conclude that our temperature determinations based on high-quality data, state-of-the-art spectral synthesis in NLTE and several independent temperature indicators are reliable and almost free of large remaining systematic effects. If adopting significantly different values in the higher temperature domain, like suggested by previous studies, the ionization equilibrium of no species will be matched any longer, hence the global and detailed observed spectra will not be reproduced by the synthetic model. We recommend, when possible, to perform a parameter determination like the full spectroscopic method presented by us in previous works (e.g. Paper I) or by Simón-Díaz (2010). If the observed spectra or the spectral synthesis are not available, a good parameter estimation can be provided by our scales presented in Section 4 (see also Nieva 2013). For $T_{\text {eff }} \leq 24000 \mathrm{~K}$, the scale $T_{\mathrm{N} 93}$ by Napiwotzki et al. (1993) also renders reliable temperatures, and for $T_{\text {eff }} \leq 21000 \mathrm{~K}$, the scales by Daflon et al. (1999) and Lyubimkov et al. (2002) also give reliable results (note the lower limit indicated by each scale). 


\section{Summary and conclusions}

Based on comprehensive previous studies which lead us to determine effective temperatures and surface gravities of 30 OB-type (O9-B3 V-III) stars at high accuracy and precision, here we investigated whether such parameters can be used to propose new calibrations to some photometric indices. The spectroscopic parameters were derived via multiple ionization equilibria and simultaneous fits to the Balmer lines based on our most recent spectrum synthesis for NLTE computations and high-quality observed spectra. We propose very useful relationships between atmospheric parameters and various photometric indices from the Johnson and the Strömgren systems. Effective temperatures can be estimated at a precision of $\sim 400 \mathrm{~K}$ for luminosity classes III/IV and $\sim 800 \mathrm{~K}$ for luminosity class V. And surface gravities can reach internal uncertainties as low as $\sim 0.08$ dex when using our calibration to the Johnson $Q$-parameter. Similar precision is achieved for gravities derived from the $\beta$-index and the precision decreases for both atmospheric parameters when using the Strömgren indices $\left[c_{1}\right]$ and $[u-b]$. Our uncertainties are smaller than typical differences to results from other methods in the literature, that reach values up to $\pm 2000 \mathrm{~K}$ for $T_{\text {eff }}$ and \pm 0.25 dex for $\log g$ typically, and in extreme cases $+6000 \mathrm{~K}$ and \pm 0.4 dex, respectively.

\section{References}

Briquet, M., Aerts, C., Baglin, A., et al., 2011, A\&A, 527, 112 (Paper III)

Butler, K., \& Giddings, J.R., 1985, Newsletter of Analysis of Astronomical Spectra, No. 9 (University College London)

Cunha, K., \& Lambert, D.L., 1992, ApJ, 399, 586

Daflon, S., Cunha, K., \& Becker, S.R., 1999, ApJ, 522, 950

Giddings, J.R., 1981, Ph.D. Thesis (Univ. London)

Kilian, J., Becker, S.R., Gehren, T., \& Nissen, P.E., 1991, A\&A, 244, 419

Kurucz, R.L., 1993a, CD-ROM No. 2-12 (SAO, Cambridge, Mass.)

Kurucz, R.L., 1993b, CD-ROM No. 13 (SAO, Cambridge, Mass.)

Lyubimkov, L.S., Rachkovskaya, T.M., Rostopchin, S.I., et al., 2002, MNRAS, 333, 9

Morel, T., \& Butler, K., 2008, A\&A, 487, 307

Napiwotzki, R., Schönberner, D., \& Wenske, V., 1993, A\&A, 268, 653

Nieva, M.F., 2007, Ph.D. Thesis (Univ. Erlangen-Nuremberg \& Observatório Nacional, Brazil)

Nieva, M.F., 2013, A\&A, 550, 26

Nieva, M.F., \& Przybilla, N., 2007, A\&A, 467, 295

Nieva, M.F., \& Przybilla, N., 2008, A\&A, 481, 199

Nieva, M.F., \& Przybilla, N., 2012, A\&A, 539, A143 (Paper I)

Nieva, M.F., \& Simón-Díaz, S., 2011, A\&A, 532, 2 (Paper II)

Przybilla, N., Nieva, M.F., \& Butler, K., 2008, ApJ, 688, L103

Przybilla, N., Nieva, M.F., \& Butler, K., 2011, JPhCS, 328, 012015

Puls, J., Urbaneja, M., Venero, R., et al., 2005, A\&A, 435, 669

Simón-Díaz, S., 2010, A\&A, 510, A22 